\title{
Composition and Diversity of CRISPR-Cas13a Systems in the Genus Leptotrichia
}

\begin{abstract}
Shinya Watanabe ${ }^{1}$, Bintao Cui ${ }^{1}$, Kotaro Kiga ${ }^{1}$, Yoshifumi Aiba ${ }^{1}$, Xin-Ee Tan', Yusuke Sato'o', Moriyuki Kawauchi', Tanit Boonsiri', Kanate Thitiananpakorn', Yusuke Taki', Fen-Yu Li' ${ }^{1}$, Aa Haeruman Azam ${ }^{1}$, Yumi Nakada ${ }^{2}$, Teppei Sasahara' ${ }^{1}$ and Longzhu Cui ${ }^{1 *}$
\end{abstract}

'Division of Bacteriology, Department of Infection and Immunity, Faculty of Medicine, Jichi Medical University, Tochigi, Japan, ${ }^{2}$ Division of Clinical Laboratory, Tottori University Hospital, Tottori, Japan

\section{OPEN ACCESS}

Edited by:

Rakesh Sharma, Institute of Genomics and Integrative

Biology (CSIR), India

Reviewed by:

Yinhua Lu,

Shanghai Normal University, China

Jose Antonio Ibarra,

National Polytechnic Institute, Mexico

*Correspondence:

Longzhu Cui

longzhu@jichi.ac.jp

Specialty section:

This article was submitted to

Evolutionary and Genomic

Microbiology,

a section of the journal

Frontiers in Microbiology

Received: 19 July 2019 Accepted: 22 November 2019 Published: 10 December 2019

Citation:

Watanabe S, Cui B, Kiga K, Aiba Y, Tan X-E, Sato'o Y,

Kawauchi $M$, Boonsiri T, Thitiananpakorn K, Taki Y, Li F-Y, Azam AH, Nakada Y, Sasahara T and

Cui L (2019) Composition and Diversity of CRISPR-Cas 13a Systems in the Genus Leptotrichia.

Front. Microbiol. 10:2838. doi: 10.3389/fmicb.2019.02838
Clustered regularly interspaced short palindromic repeats (CRISPR)-Cas13a, previously known as CRISPR-C2c2, is the most recently identified RNA-guided RNA-targeting CRISPR-Cas system that has the unique characteristics of both targeted and collateral single-stranded RNA (ssRNA) cleavage activities. This system was first identified in Leptotrichia shahii. Here, the complete whole genome sequences of 11 Leptotrichia strains were determined and compared with 18 publicly available Leptotrichia genomes in regard to the composition, occurrence and diversity of the CRISPR-Cas13a, and other CRISPR-Cas systems. Various types of CRISPR-Cas systems were found to be unevenly distributed among the Leptotrichia genomes, including types I-B (10/29, 34.4\%), II-C (1/29, 2.6\%), III-A (6/29, 15.4\%), III-D (6/29, 15.4\%), III-like (3/29, 7.7\%), and VI-A (11/29, 37.9\%), while 8 strains (20.5\%) had no CRISPR-Cas system at all. The Cas13a effectors were found to be highly divergent with amino acid sequence similarities ranging from $61 \%$ to $90 \%$ to that of $L$. shahii, but their collateral ssRNA cleavage activities leading to impediment of bacterial growth were conserved. CRISPR-Cas spacers represent a sequential achievement of former intruder encounters, and the retained spacers reflect the evolutionary phylogeny or relatedness of strains. Analysis of spacer contents and numbers among Leptotrichia species showed considerable diversity with only $4.4 \%$ of spacers (40/889) were shared by two strains. The organization and distribution of CRISPR-Cas systems (type I-VI) encoded by all registered Leptotrichia species revealed that effector or spacer sequences of the CRISPR-Cas systems were very divergent, and the prevalence of types I, III, and VI was almost equal. There was only one strain carrying type II, while none carried type IV or $\mathrm{V}$. These results provide new insights into the characteristics and divergences of CRISPR-Cas systems among Leptotrichia species.

Keywords: Leptotrichia, CRISPR-Cas13a, clustered regularly interspaced short palindromic repeats, CRISPRCas, C2c2, crRNA, protospacer, self-targeting spacer 


\section{INTRODUCTION}

Leptotrichia species, which are strict anaerobic or facultative anaerobic Gram-negative rods found in the oral cavity, intestines, female genital tract and urogenital system of both humans and animals, can ferment carbohydrates to generate multiple products, including lactic acid, and traces of acetic, formic or succinic acid, depending on the substrates and species (Eribe and Olsen, 2017). Some Leptotrichia species cause opportunistic infectious diseases, including gingivitis, periodontitis, infectious endocarditis and bacteraemia (Eribe and Olsen, 2008, 2017). With the recent progress and spread of modern techniques, the involvement of Leptotrichia species in various infectious diseases has gained considerable attention (Eribe and Olsen, 2017). However, the exact clinical impact of Leptotrichia species relevant to infectious diseases remains unclear due to difficulties in bacterial culture, isolation and identification. To date, eight Leptotrichia species have been characterized, which include $L$. buccalis, $L$. goodfellowii, L. hofstadii, $L$. shahii, L. trevisanii, L. massiliensis, L. hongkongensis, and L. wadei. However, many clinical Leptotrichia isolates have remained unclassified due to the lack of the whole-genome sequences and biochemical properties of these isolates (Eribe and Olsen, 2017). Currently, Leptotrichia genome data are very limited as there are only one complete whole genome sequences of species-identified strain and three unidentified strains available in the GenBank database (Ivanova et al., 2009; Gupta and Sethi, 2014).

Clustered regularly interspaced short palindromic repeats (CRISPR) and CRISPR-associated genes (cas) encode proteins involved in the adaptive immune response against archaea and bacteria by preventing an invasion of the host cell by foreign genetic elements, such as bacteriophages and plasmids (Marraffini, 2015). The CRISPR-Cas systems identified thus far show extreme diversity in cas gene compositions as well as genomic loci architecture. Despite this diversity, the latest classification system categorizes the CRISPR-Cas systems into two classes based on basic compositions and structures: class 1 systems, consisting of types I and III as well as the putative type IV, which possess multi-subunit effector complexes comprised of multiple Cas proteins, and class 2 systems, which are comprised of types II, V, and VI that are characterized by effector complexes consisting of a single, large Cas protein (Makarova et al., 2018; Shmakov et al., 2018). CRISPR-Cas13a (known previously as CRISPR-C2c2) is the most recently identified CRISPR-Cas system belonging to the type VI class 2 system and is characterized by cleavage activity of a single RNAguided single-stranded RNA (ssRNA) molecule (Shmakov et al., 2015). An interesting finding recently made by Abudayyeh et al. (2016) is that CRISPR-Cas13a of L. shahii can perform promiscuous collateral ssRNA cleavage. The same research group and others recently demonstrated that the CRISPR-Cas13a system is an efficient molecular tool for the cleavage and editing of target RNA in both prokaryotic and mammalian cells (Abudayyeh et al., 2016, 2017; Cox et al., 2017), with highly sensitive detection of nucleic acids (East-Seletsky et al., 2017; Gootenberg et al., 2017).
The genus Leptotrichia is the main source of the CRISPRCas13a system (Shmakov et al., 2015). While the L. shahii CRISPR-Cas13a system has been intensively studied, the variations in the genetic features of CRISPR-Cas systems demonstrated so far have prompted us to carry out this comprehensive study of the occurrence, composition and diversity of CRISPR-Cas13a, and other CRISPR-Cas systems in the genus Leptotrichia.

\section{MATERIALS AND METHODS}

\section{Leptotrichia Strains and Growth Conditions}

Three Leptotrichia trevisanii strains, JMUB3870, JMUB3935 and JMUB4039, two L. wadei strains, JMUB3933 and JMUB3934 and one L. hongkongensis strain, JMUB5056, were isolated between 2012 and 2019 from blood cultures of patients in Japan. Leptotrichia strain JMUB3936 was isolated in 2017 from the bronchial lavage fluid of a patient in Japan. The following type strains of Leptotrichia species were obtained from the Japan Collection of Microorganisms (JCM): L. goodfellowii JCM16774, L. hofstadii JCM16775, L. shahii JCM16776 and L. wadei JCM16777. Leptotrichia strains were stored in Gifu Anaerobic Medium (GAM broth; Nissui Pharmaceutical Co., Ltd., Tokyo, Japan) supplemented with $40 \%$ glycerol at $-80^{\circ} \mathrm{C}$, and were recovered for the present study on various agar plates, of which isolates JCM12969, JCM16774, JMUB3933, JMUB3934, JMUB3935, JMUB4039, and JMUB3870 were inoculated on GAM agar, isolates JCM16775, JCM16776, JCM16777, and JMUB3936 were recovered on sheep blood agar (Kohjin Bio Co., Ltd., Saitama, Japan), and isolates JMUB5056 was grown on Anaero Columbia Agar with Rabbit Blood (Nippon Becton Dickinson Company, Ltd., Tokyo, Japan). All plates were incubated anaerobically under conditions of $5 \% \mathrm{CO}_{2}$ with an oxygen absorbent (Anaeropack Kenki, Mitsubishi Gas Chemical Company, Tokyo, Japan) at $37^{\circ} \mathrm{C}$ for 48-72 h. Escherichia coli M1601 cells were grown in Luria-Bertani (LB) medium (Nippon Becton Dickinson Company, Ltd., Tokyo, Japan) with shaking or on LB agar with antibiotic supplementation (chloramphenicol at $10 \mu \mathrm{g} / \mathrm{mL}$; kanamycin at $50 \mu \mathrm{g} / \mathrm{mL}$ ) as needed for plasmid maintenance.

\section{Whole-Genome Sequencing and Sequence Read Assembly}

Bacterial genomic DNA was extracted and purified using the NucleoBond AXG kit (Takara Bio, Inc., Otsu, Shiga, Japan). DNA library preparation was performed using a Rapid Barcoding sequencing kit (SQK-RBK004; Oxford Nanopore Technologies, Oxford, United Kingdom). Sequencing was then performed using a MinION Mk-1B device integrated with a FLO-MIN-106 flow cell (Oxford Nanopore Technologies). Base calling was carried out using MinKNOW software (Oxford Nanopore Technologies) and the Porechop tool 
(version $0.2 .4^{1}$ ) was used for demultiplexing of barcoded libraries. The resulting sequence reads were assembled with the Canu tool (version 1.8 ${ }^{2}$ ) (Koren et al., 2017). The assembled genomes were then circularized using the Circlator tool (version 1.5.5 ) (Hunt et al., 2015), and all of the resulting assemblies that produced contiguous sequences were polished with the nanopolish algorithm (version $0.11 .0^{4}$ ) (Loman et al., 2015). Genome sequencing of all of the above strains was performed using the MiSeq platform as previously described (Watanabe et al., 2016, 2018). The draftgenomes generated by MinION were then polished again with the reads generated by MiSeq with the Pilon automated genome assembly improvement and variant detection tool (version 1.22; Broad Institute, Cambridge, MA, United States) (Walker et al., 2014). The resulting genome sequences were further trimmed using the CLC genomics workbench (Qiagen, Hilden, Germany).

\section{Gene Annotation and Genome Analysis}

Gene extraction and annotation were performed with the Microbial Genome Annotation Pipeline ${ }^{5}$ or Prokka (version $1.13 .3^{6}$ ) (Seemann, 2014). CRISPR-Cas genes were extracted and annotated using the CRISPRCasFinder program ${ }^{7}$ (Couvin et al., 2018). Prophages were identified by PHASTER ${ }^{8}$ (Arndt et al., 2016). The kSNP trees were constructed with the kSNP alignment-free sequence analysis tool (version 3.021) (Gardner et al., 2015) and then visualized with the FigTree graphical viewer of phylogenetic trees (version $1.4 .3^{9}$ ). Whole-genome average nucleotide identity (ANI) was calculated by FastANI and the heat map was depicted by ggplot2 of R (Jain et al., 2018). Pairwise blastn analysis for CRISPR-cas loci was carried out using the Easyfig application (version 2.2.2 $2^{10}$ ) (Sullivan et al., 2011).

\section{Plasmid Construction}

To examine the collateral growth inhibition activities of various Cas13a loci of Leptotrichia species, two sets of vectors were constructed. One set was for the expression of CRISPR-Cas13a and the other for the generation of their target RNA. The original plasmid pC003 containing a L. shahii Cas13a locus. First, the native Cas13a loci including native direct repeats and spacers were isolated from the genome of each Leptotrichia strain by polymerase chain reaction (PCR) and then cloned into the pC003 vector by replacing the shahiiCas13a locus using the In-Fusion HD Cloning Kit (Takara Bio, Inc., Shiga, Japan), and then kanamycin resistant gene of the vector was replaced by kanamycin-resistant gene $(a p h A-3)$ to generate

\footnotetext{
${ }^{1}$ https://github.com/rrwick/Porechop

${ }^{2} \mathrm{http}: / /$ canu.readthedocs.org/

${ }^{3} \mathrm{http}: / /$ sanger-pathogens.github.io/circlator/

${ }^{4}$ https://github.com/jts/nanopolish

${ }^{5} \mathrm{http}: / /$ www.migap.org

${ }^{6} \mathrm{https} / / /$ github.com/tseemann/prokka

${ }^{7}$ https://crisprcas.i2bc.paris-saclay.fr/

${ }^{8} \mathrm{http}: / /$ phaster.ca/

${ }^{9} \mathrm{http}: / /$ tree.bio.ed.ac.uk/software/figtree/

${ }^{10} \mathrm{http}: / /$ mjsull.github.io/Easyfig/
}

pKLC-Cas13a. The pC003-LshC2C2 locus included in the plasmid pACYC184 for spacer cloning was a gift from Dr. Feng Zhang (Addgene plasmid \#79152 ${ }^{11}$; RRID:Addgene_79152) (Abudayyeh et al., 2016).

To generate arabinose-inducible target RNA expression vector, firstly we constructed arabinose-inducible RFP expression vector (pKLC-RFP) from pC008-pBR322 by replacing tetracycline-inducible element (pBAD33) and ampicillin resistant gene $(b l a)$ to arabinose-inducible element and chloramphenicol acetyltransferase (CAT). Then the target sequence of the native Cas13a was inserted into just behind the RFP coding sequence. A native spacer sequence from each Leptotrichia strain and the protospacer adjacent motif, nucleotide $\mathrm{C}$ in this study, were adapted to the $5^{\prime}$ end of the reverse vector primer. The final plasmid backbone was generated by PCR followed by self-ligation using the In-Fusion HD Cloning Kit. All of the generated plasmids were confirmed by Sanger sequencing. The pC008-pBR322 with tetR-inducible RFP was a gift from Dr. Feng Zhang (Addgene plasmid \# 79157212 RRID:Addgene_79157). The primers used in this study are listed in Supplementary Table S1.

\section{Cas13a Collateral Growth Inhibition Assay}

The assay for E. coli growth inhibition as a result of collateral ssRNA cleavage by Cas13a was carried out based on the previous paper (Abudayyeh et al., 2016). The constructed plasmids were chemically transformed into competent E. coli MC1601 cells. Briefly, $500 \mathrm{ng}$ of CRISPR-Cas13a expression plasmid and $100 \mathrm{ng}$ of arabinose-inducible target plasmid were mixed and added to the competent cells obtained above. Then, the transformation reaction was incubated on ice for $30 \mathrm{~min}$, followed by heat shock at $42^{\circ} \mathrm{C}$ in a heat block for exactly $45 \mathrm{~s}$, and then immediately transferred to tubes containing $1 \mathrm{~mL}$ of $\mathrm{LB}$ broth at room temperature, which were cooled on ice. Afterward, the samples were incubated at $37^{\circ} \mathrm{C}$ for $1 \mathrm{~h}$ to induce antibiotic resistance and then plated on LB agar plates containing kanamycin and chloramphenicol at $37^{\circ} \mathrm{C}$ until colonies appeared. Then, the colonies were transferred to the LB medium containing kanamycin and chloramphenicol and incubated with gentle shaking until early stationary phase. The optical density at $600 \mathrm{~nm}$ (OD 600) of the bacterial culture was adjusted to 0.1 with LB medium containing kanamycin and chloramphenicol, and then $100 \mu \mathrm{l}$ of the medium were transferred to L-shaped shaking tubes containing $10 \mathrm{ml}$ of LB medium with kanamycin, chloramphenicol and $0.2 \% \mathrm{~L}$-arabinose to induce target gene expression. During the incubation at $25 \mathrm{rpm}$ at $37^{\circ} \mathrm{C}$, the optical density at $600 \mathrm{~nm}$ of the bacterial culture were measured every 5 min by TVS126MB (ADVANTEC Toyo Kaisha, Ltd.) for at least $12 \mathrm{~h}$. The Cas13a collateral growth inhibition assay was performed in biological triplicate.

\section{Accession of the Genome Sequence}

The genome sequences were deposited to the DNA Data Bank of Japan (DDBJ, Bioproject accession number PRJDB7856) and the

\footnotetext{
${ }^{11}$ http://n2t.net/addgene:79152

${ }^{12}$ http://n2t.net/addgene:79157
} 
sequence raw data were deposited in the DDBJ Sequence Read Archive (DRA007809).

\section{RESULTS AND DISCUSSION}

\section{Comparative Analysis of Leptotrichia Genome}

CRISPR-Cas13a systems are broadly distributed among Leptotrichia species. The estimation of the occurrence, composition, and diversity of CRISPR-Cas13a systems in the genus Leptotrichia is particularly challenging because the complete whole-genome information is limited. At present, the genus Leptotrichia is composed of eight species. However, the complete whole genome sequences are not available except for L. buccalis (Ivanova et al., 2009) and three unidentified Leptotrichia strains. In this study, 11 strains of six Leptotrichia species were collected, which included L. goodfellowii, L. hofstadii, L. hongkongensis, L. shahii, L. trevisanii and L. wadei, and their complete whole-gnome sequences were determined (Supplementary Table S2). As it was not possible to collect isolates of the remaining species, a draft genome of $L$. massiliensis available from a public database was used. Taken together with the additional draft genome sequences retrieved from the public database, a total of 29 whole-genome sequences were used for comparative genomic analysis. All genomes were surveyed and investigated for the composition, occurrence and diversity of the CRISPR-Cas13a and other CRISPR-Cas systems. The overall features of the genomes, the distribution of the CRISPR-Cas systems, and the numbers of CRISPR-Cas spacers, plasmids and prophages for each strain are summarized in Table 1. As shown in Table 1, the chromosome size of the genus Leptotrichia varies from 2,142,946 to 2,829,322 bp with GC contents of $29.5 \%$ to $31.7 \%$. Virulence factors were searched against all genomes using Prokka (version 1.13.3), but no class of genes was found to contribute to bacterial pathogenesis.

To depict the relationship among Leptotrichia species and strains, a whole-genome single-nucleotide polymorphism (SNP)based phylogenetic tree was constructed of a total of 29 Leptotrichia genomes, which included the complete whole genome-sequencing data determined in the present study. As shown in Figure 1, four sequenced Leptotrichia type strains (i.e., L. goodfellowii JCM16774, L. hofstadii JCM16775, L. shahii JCM16776, and L. wadei JCM16777) belonged to the same clade of the corresponding strains registered as The Department of Energy (DOE) draft-genomes (i.e., L. goodfellowii DSM 19756, L. hofstadii DSM 21651, L. shahii DSM 19757, and L. wadei DSM 19758). The analysis also revealed that except for strain JMUB3936 which was originally classified into $L$. wadei by the biochemical property in a clinical microbiology laboratory, all strains were classified into corresponding clades. To further elucidate the species classification of the Leptotrichia strains, we performed the whole-genome ANI analysis showing the average nucleotide identities between all orthologous genes shared between the two genomes (Figure 2 and Supplementary Table S3). ANI revealed that the 29 Leptotrichia genomes were classified into 14 species including six unclassified species because bacterial strains belonging to the same species typically showing $\geq 95 \%$ ANI among themselves (Konstantinidis and Tiedje, 2005; Goris et al., 2007). Based on the ANI analysis, strain JMUB3936 was classified into new species because the highest ANI was 92.2\% (L. wadei JMUB3933). Strain JMUB3936 was located between L. wadei and L. shahii. In addition, $16 \mathrm{~S}$ rRNA sequence analysis of strain JMUB3936 showed $97.6 \%$ and $96.1 \%$ identities to those of L. wadei type strain DSM19758 and L. shahii type strain DSM19757, supporting that JMUB3936 must be classified into a new species of genus Leptotrichia. 16S rRNA sequence analysis is one of the gold standards classifying species and genus, and two isolates having $16 \mathrm{~S}$ rRNA sequence similarities of $\leq 99 \%$ can generally be classified into different species in the same genus (Clarridge, 2004). Furthermore, JMUB3936 can be considered to be a member of human oral or skin microbiota, since all but one out of $6216 \mathrm{~S}$ rRNA sequences came from samples of human oral or skin microbiota deposited in NCBI database (Munson et al., 2004; Täubel et al., 2009; Bik et al., 2010; Bolivar et al., 2012; Kong et al., 2012) had high similarities to that of JMUB3936 ( $\geq 99 \%$ identity with $\geq 80 \%$ coverage).

To date, eight species of Leptotrichia have been precisely described (Eribe et al., 2004; Woo et al., 2010). Apart from the eight Leptotrichia species, previous analysis based on the 16S rRNA gene identified 10 unclassified Leptotrichia species, indicating that some Leptotrichia strains have not yet being assigned a species name (Eribe and Olsen, 2008, 2017). Species classification based on both phenotype and genotype is an essential starting point to understand the biology, ecology and clinical impacts of Leptotrichia species.

\section{CRISPR-Cas Systems in Leptotrichia}

To investigate the composition, occurrence and diversity of CRISPR-Cas systems among Leptotrichia species, all 29 Leptotrichia genomes were analyzed for the presence of CRISPRs and cas genes with the use of the CRISPRCasFinder program, which enables the identification of direct repeat consensus boundaries and the extraction of related spacers and cas genes (Couvin et al., 2018). This analysis identified a total of 38 CRISPR-Cas systems: 10 (26.3\%) type I-B, 1 (2.6\%) type II-C, $6(15.8 \%)$ type III-A, 6 (15.8\%) type III-D, 3 (7.9\%) type IIIlike and $12(31.6 \%)$ type VI-A, while no type IV and V systems were detected. Of the 29 strains, 8 (27.6\%) had no CRISPRCas system (Table 1). Hence, we concluded that types I, III, and VI were the most dominant CRISPR-Cas systems among Leptotrichia species.

Illustrations of all CRISPR-Cas systems identified in this study are summarized in Figure 3. Type I-B CRISPR-Cas systems were distributed in five known and one unclassified Leptotrichia species (Figure 3A). It is well known that CRISPR mediates adaptive immunity according to the following three steps: (1) acquisition of short segments of foreign nucleic acids into CRISPR loci (adaptation); (2) crRNA expression and maturation (crRNA-processing); (3) target interference by recognition of the crRNA and destruction of the target nucleic acid (interference) (Bhaya et al., 2011). The Leptotrichia type I-B CRISPR-cas loci were found to include full gene sets responsible for the above 
TABLE 1 | Genome and CRISPR-Cas system information of genus Leptotrichia.

\begin{tabular}{|c|c|c|c|c|c|c|c|c|c|c|c|c|c|c|}
\hline \multicolumn{6}{|c|}{ Genome infromation } & \multicolumn{9}{|c|}{ CRISPR/Cas class } \\
\hline Species & Strain & $\begin{array}{l}\text { Genome sequencing } \\
\text { type }^{\mathrm{b}}\end{array}$ & $\begin{array}{l}\text { Chromosome } \\
\text { length (bp) }\end{array}$ & GC $\%$ & $\begin{array}{l}\text { GenBank } \\
\text { accession no. }\end{array}$ & $\begin{array}{l}\text { Number of } \\
\text { plasmid }\end{array}$ & $\begin{array}{l}\text { Namber of } \\
\text { prophage/ } \\
\text { genomic } \\
\text { island }\end{array}$ & 1 & II & III & IV & $\mathbf{v}$ & VI & $\begin{array}{l}\text { Number of } \\
\text { spacerc }\end{array}$ \\
\hline L. wadei & KA00185 & Draft genome & $n / a^{a}$ & n/a & GCA_001553045.1 & $n / a$ & $n / a$ & - & - & - & - & - & - & 5 \\
\hline L. wadei & JMUB3933 & Complete genome & 2361227 & $29.6 \%$ & AP019834 & 0 & 2 & I-B & - & - & - & - & VI-A1, VI-A2 & 47 \\
\hline L. wadei & JMUB3934 & Complete genome & 2414633 & $29.6 \%$ & AP019835 & 4 & 2 & - & - & - & - & - & VI-A & 1 \\
\hline L. wadei & F0279 & Draft genome & $n / a$ & $n / a$ & GCA_000469405.1 & $\mathrm{n} / \mathrm{a}$ & $\mathrm{n} / \mathrm{a}$ & - & - & - & - & - & VI-A & 7 \\
\hline L. wadei & JCM16777 & Complete genome & 2305216 & $29.5 \%$ & AP019829 & 1 & 0 & - & - & - & - & - & - & 3 \\
\hline L. wadei & DSM 19758 & Draft genome & $\mathrm{n} / \mathrm{a}$ & $n / a$ & GCA_000373345.1 & $\mathrm{n} / \mathrm{a}$ & $\mathrm{n} / \mathrm{a}$ & - & - & - & - & - & - & 3 \\
\hline Leptotrichia sp.-1 & JMUB3936 & Complete genome & 2335974 & $30.1 \%$ & AP019841 & 3 & 1 & - & - & III-A1, III-A2 & - & - & - & 22 \\
\hline L. shahii & DSM 19757 & Draft genome & $\mathrm{n} / \mathrm{a}$ & $n / a$ & GCA_000373045.1 & $\mathrm{n} / \mathrm{a}$ & $\mathrm{n} / \mathrm{a}$ & I-B & - & III-A & - & - & VI-A & 51 \\
\hline L. shahii & JCM16776 & Complete genome & 2142946 & $29.7 \%$ & AP019827 & 1 & 1 & I-B & - & III-A & - & - & VI-A & 63 \\
\hline Leptotrichia sp.-2 & F0557 & Draft genome & $\mathrm{n} / \mathrm{a}$ & $n / a$ & GCA_000469385.1 & $\mathrm{n} / \mathrm{a}$ & $\mathrm{n} / \mathrm{a}$ & - & - & - & - & - & VI-A & 6 \\
\hline L. hongkongensis & JMUB5056 & Complete genome & 2261073 & $29.9 \%$ & AP019846 & 1 & 1 & - & - & - & - & - & - & 11 \\
\hline L. massiliensis & P3007 & Draft genome & $\mathrm{n} / \mathrm{a}$ & $\mathrm{n} / \mathrm{a}$ & GCA_900104625.1 & $\mathrm{n} / \mathrm{a}$ & $\mathrm{n} / \mathrm{a}$ & - & - & - & - & - & VI-A1, VI-A2 & 21 \\
\hline L. massiliensis & F0581 & Draft genome & $n / a$ & $n / a$ & GCA_000469525.1 & $\mathrm{n} / \mathrm{a}$ & $\mathrm{n} / \mathrm{a}$ & - & - & - & - & - & VI-A & 10 \\
\hline L. hofstadii & JCM16775 & Complete genome & 2548198 & $30.6 \%$ & AP019823 & 3 & 1 & - & - & III-A & - & - & - & 10 \\
\hline L. hofstadii & DSM 21651 & Draft genome & $\mathrm{n} / \mathrm{a}$ & $n / a$ & GCA_000428965.1 & $\mathrm{n} / \mathrm{a}$ & $\mathrm{n} / \mathrm{a}$ & - & - & III-A & - & - & - & 6 \\
\hline L. hofstadii & F0254 & Draft genome & $\mathrm{n} / \mathrm{a}$ & $\mathrm{n} / \mathrm{a}$ & GCA_000162955.1 & $\mathrm{n} / \mathrm{a}$ & $\mathrm{n} / \mathrm{a}$ & - & - & - & - & - & - & 0 \\
\hline L. trevisanii & DSM 22070 & Draft genome & $\mathrm{n} / \mathrm{a}$ & $\mathrm{n} / \mathrm{a}$ & GCA_000482505.1 & $\mathrm{n} / \mathrm{a}$ & $\mathrm{n} / \mathrm{a}$ & I-B & - & $|1|-D$ & - & - & - & 17 \\
\hline L. trevisanii & JMUB3870 & Complete genome & 2829322 & $30.6 \%$ & AP019831 & 2 & 1 & I-B & - & $\| 11-D$ & - & - & - & 78 \\
\hline L. trevisanii & JMUB4039 & Complete genome & 2685755 & $30.8 \%$ & AP019845 & 0 & 1 & I-B & - & |II-D & - & - & VI-A & 56 \\
\hline L. trevisanii & JMUB3935 & Complete genome & 2729392 & $30.6 \%$ & AP019840 & 0 & 2 & - & - & |II-D & - & - & - & 14 \\
\hline L. buccalis & C-1013-b & Complete genome & 2465610 & $29.6 \%$ & GCA_000023905.1 & 0 & 0 & I-B & - & |II-D & - & - & VI-A & 102 \\
\hline Leptotrichia sp.-3 & F0260 & Complete genome & 2194935 & $29.8 \%$ & GCA_001553645.1 & 0 & 2 & - & - & - & - & - & - & 1 \\
\hline Leptotrichia sp.-4 & bin_23 & Draft genome & $\mathrm{n} / \mathrm{a}$ & $\mathrm{n} / \mathrm{a}$ & GCA_003638725.1 & $\mathrm{n} / \mathrm{a}$ & $\mathrm{n} / \mathrm{a}$ & - & - & - & - & - & - & 0 \\
\hline Leptotrichia sp.-4 & F0590 & Complete genome & 2152181 & $29.6 \%$ & GCA_002240055.1 & 0 & 1 & - & - & $|1|-D$ & - & - & - & 37 \\
\hline L. goodfellowii & F0264 & Draft genome & $\mathrm{n} / \mathrm{a}$ & $n / a$ & GCA_000176335.1 & $\mathrm{n} / \mathrm{a}$ & $\mathrm{n} / \mathrm{a}$ & - & - & - & - & - & - & 11 \\
\hline L. goodfellowii & JCM16774 & Complete genome & 2290729 & $31.7 \%$ & AP019822 & 0 & 1 & I-B & - & III-like & - & - & - & 38 \\
\hline L. goodfellowii & DSM 19756 & Draft genome & $n / a$ & $n / a$ & GCA_000516535.1 & $\mathrm{n} / \mathrm{a}$ & $\mathrm{n} / \mathrm{a}$ & I-B & - & III-like & - & - & - & 39 \\
\hline Leptotrichia sp.-5 & W9775 & Draft genome & $\mathrm{n} / \mathrm{a}$ & $n / a$ & GCA_000469505.1 & $\mathrm{n} / \mathrm{a}$ & $\mathrm{n} / \mathrm{a}$ & - & $\|-\mathrm{C}$ & - & - & - & - & 19 \\
\hline Leptotrichia sp.-6 & W10393 & Complete genome & 2444904 & $31.4 \%$ & GCA_001274535.1 & 0 & 0 & I-B & - & III-like & - & - & - & 201 \\
\hline
\end{tabular}

${ }^{a}$ n/a indicates not applicable; ${ }^{b}$ Bold font indicates that genome sequences were determined in this study; ${ }^{c}$ Total number of spacer carried by all types of CRISPR-Cas systems. 


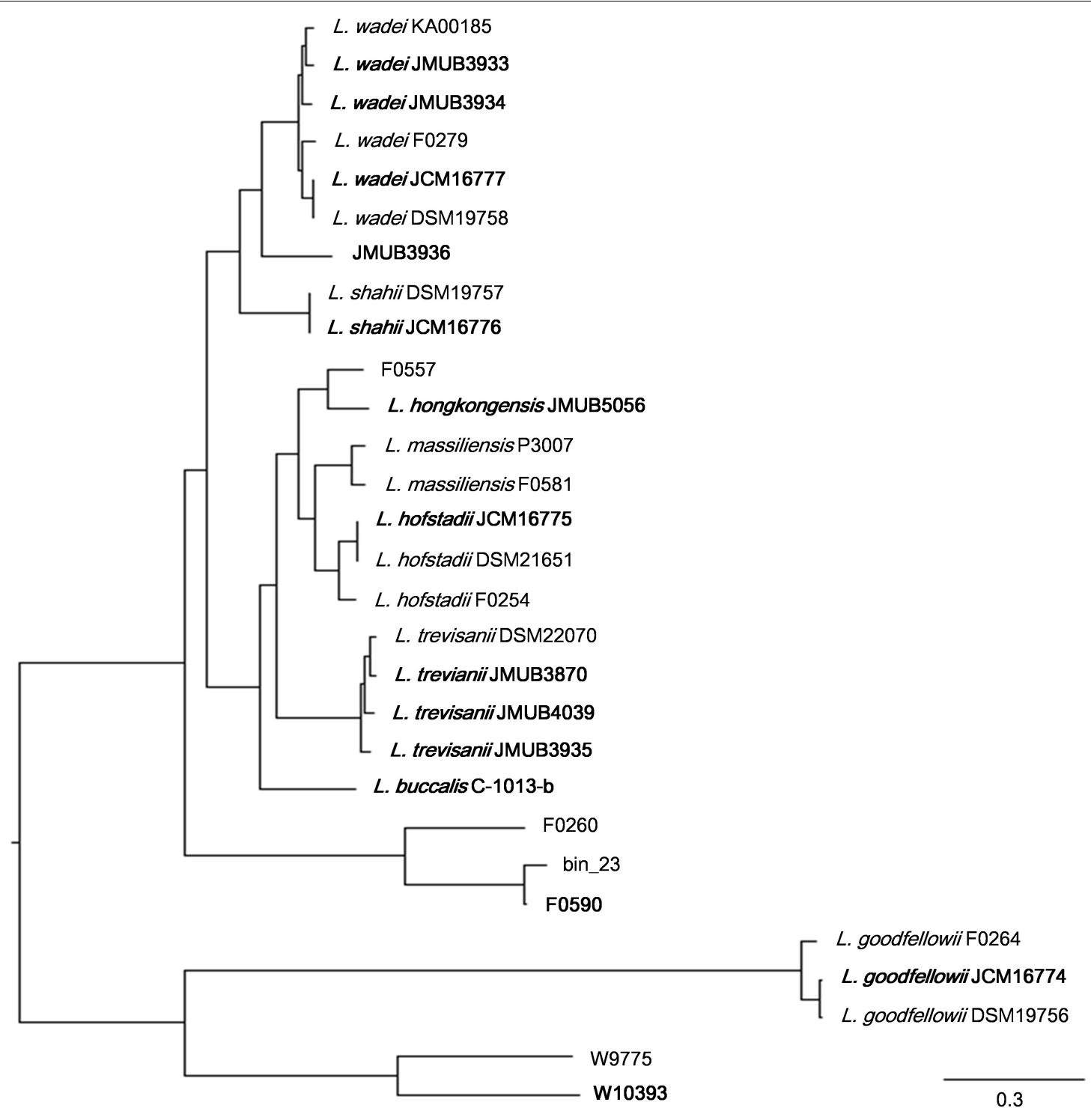

FIGURE 1 | Whole genome SNP-based majority phylogenetic tree of 29 Leptotrichia strains generated by kSNA3.0. Horizontal branch lengths indicate changes per number of SNPs. Strains in boldface are the strains their whole genomes were determined in this study. It should be noted that this is a SNPs-based (present in at least $75 \%$ strains), not an alignment based tree and no evolutionary direction can be inferred from this tree.

three steps: cas1, cas 2 and cas 4 for adaptation, cas6 for crRNAprocessing and cas3, cas5, cas7 and cas 8 for interference. All CRISPRs of type I-B CRISPR-Cas systems found in this study were located downstream of the cas 2 gene and/or upstream of the cas6 gene, which was similar with a recent report of the anaerobic rod Clostridium thermocellum by Zöphel et al. ${ }^{13}$ The type II-C CRISPR-cas system was found in only one Leptotrichia strain, W9775 (Figure 3B). Type II-C is a class 2 CRISPRCas system, and the locus contains a single effector gene (cas9) and three adaptation genes (cas1, cas2, and csn2). CRISPR was located downstream of $\operatorname{csn} 2$. Type III systems have been classified into four main subtypes (III-A to D) (Vestergaard et al., 2014;

${ }^{13}$ https://archiv.ub.uni-marburg.de/diss/z2015/0381
Makarova et al., 2015). Among the Leptotrichia genomes analyzed in this study, six type III-A, six type III-D and three type III-like loci were identified (Figure 3C). Type III systems are reported to frequently carry accessory genes either within or immediately bordering the core cas gene clusters (Haft et al., 2005; Vestergaard et al., 2014; Shah et al., 2019). The type IIIA systems of Leptotrichia can be classified into two subtypes: III-A-Lep1 and III-A-Lep2. The III-A-Lep1 loci were found in the genomes of L. hofstadii strain JCM16775 and strain JMUB3936, which carried the full set of cas genes involving the adaptation, crRNA-processing and interference steps. However, the type III-A-Lep2 system found in the genomes of L. shahii strain JCM16776 and strain JMUB3936 did not carry the csm6 gene, which encodes an HEPN family ribonuclease that 

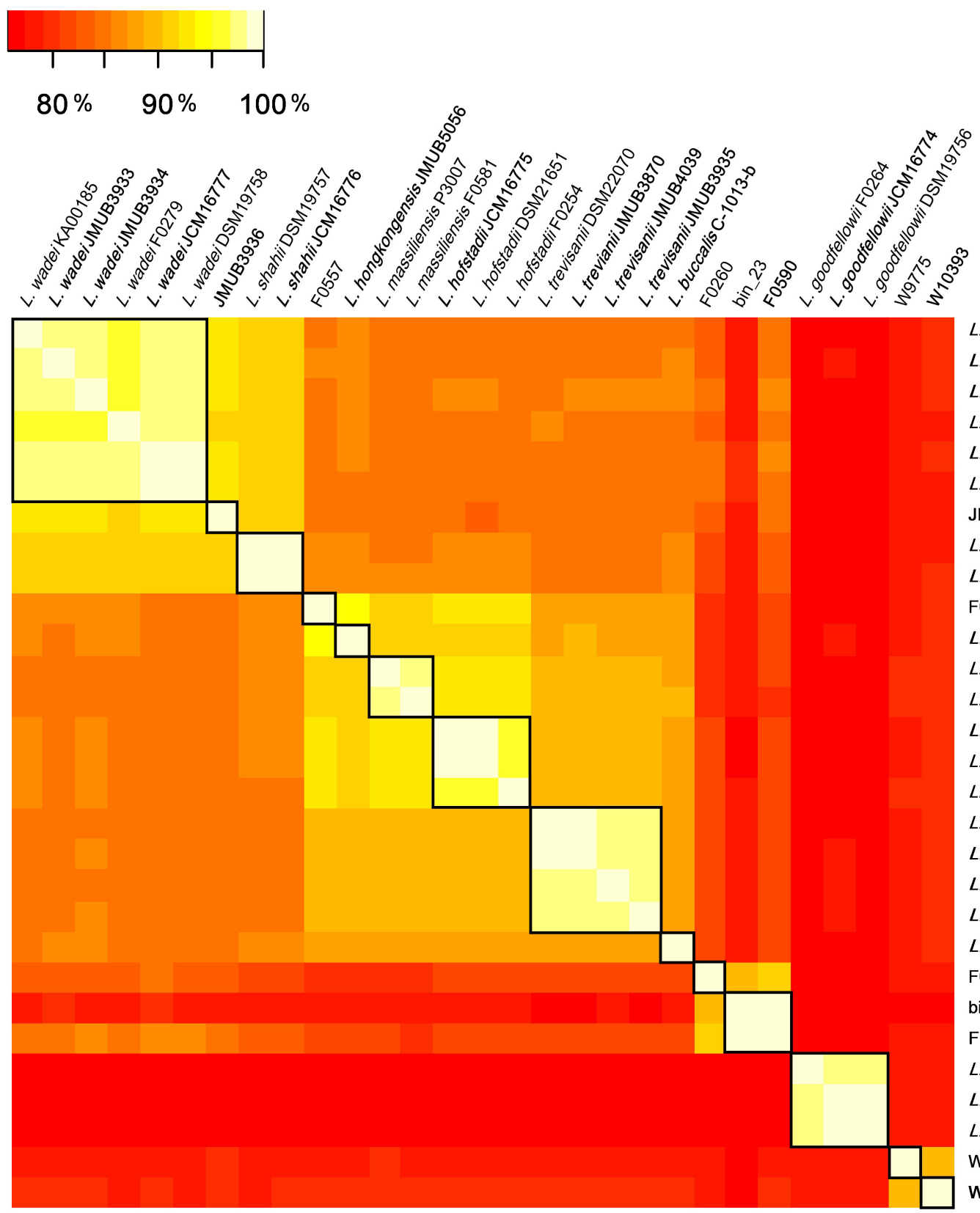

L. wadei KA00185

L. wadeiJMUB3933

L. wadeiJMUB3934

L. wadei $\mathrm{F} 0279$

L. wadeiJCM16777

L. wadei DSM19758

JMUB3936

L. shahii DSM19757

L. shahiïJCM16776

F0557

L. hongkongensis JMUB5056

L. massiliensis $\mathrm{P} 3007$

L. massiliensis $\mathrm{F} 0581$

L. hofstadii JCM16775

L. hofstadii DSM21651

L. hofstadii F0254

L. trevisanii DSM22070

L. trevianiiJMUB3870

L. trevisaniiJMUB4039

L. trevisaniiJMUB3935

L. buccalis C-1013-b

F0260

bin 23

F0590

L. goodfellowii F0264

L. goodfellowii JCM16774

L. goodfellowii DSM19756

W9775

W10393

FIGURE 2 | The whole-genome average nucleotide identity (ANI) analysis of 29 Leptotrichia genomes generated by FastANI. The genetic relatedness between two genomes was estimated by ANI analysis. $\geq 95 \%$ ANI, which generally indicates genomes belonging to the same species, was highlighted by square in black.

mediates sequence-unspecific ssRNA cleavage and contributes to anti-plasmid immunity (Niewoehner and Jinek, 2016; Foster et al., 2019). Instead of $\operatorname{csm} 6$, a $\operatorname{cs} x 1$ gene, which encodes a Csm6 homologous protein and is known to be involved in ssRNA disruption (Sheppard et al., 2016; Han et al., 2017), was identified upstream of the core cas genes in the type III-ALep2 loci.

Leptotrichia type III-D CRISPR-Cas systems were identified in four $L$. trevisanii strains, one $L$. buccalis strain and one unclassified Leptotrichia strain, F0590 (Figure 3C). The genomes of L. buccalis strains C-1031-b and F0590, had large regions of the type III-D loci, which included core cas genes, csx1, CRISPRs and putative accessory genes. Although L. trevisanii strains also have type III-D gene clusters similar to those of L. buccalis C-1031-b and L. sp. F0590, the type III-D loci were split into two clusters. In $L$. trevisanii genomes, the core cas genes involved in crRNAprocessing and interference steps were located more than $200 \mathrm{~kb}$ from the gene cluster of the accessory gene $\operatorname{cs} x 1$, adaptation genes cas4, cas2, and cas 1 and CRISPRs. We also found type III-like CRISPR-cas loci, which include csm3 genes and CRISPRs in the 
A Type I-B CRISPR-cas

\section{E Type VI-A CRISPR-cas}

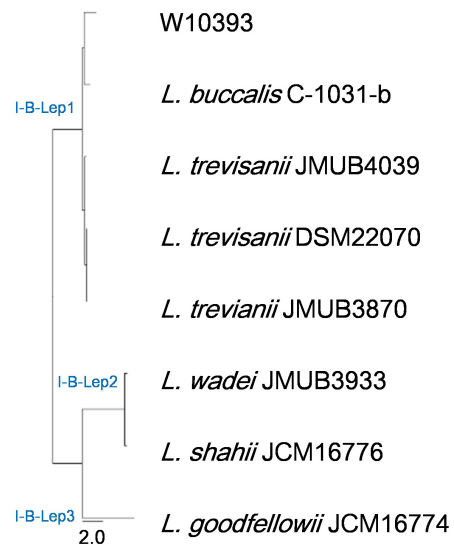

L. goodfellowii JCM16774
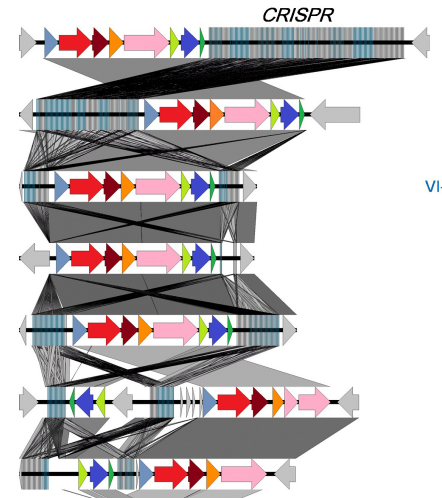

the A MAlt

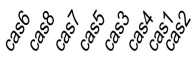

B Type II-C CRISPR-cas W9775

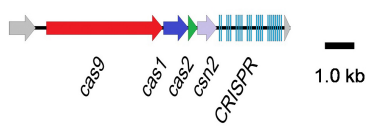

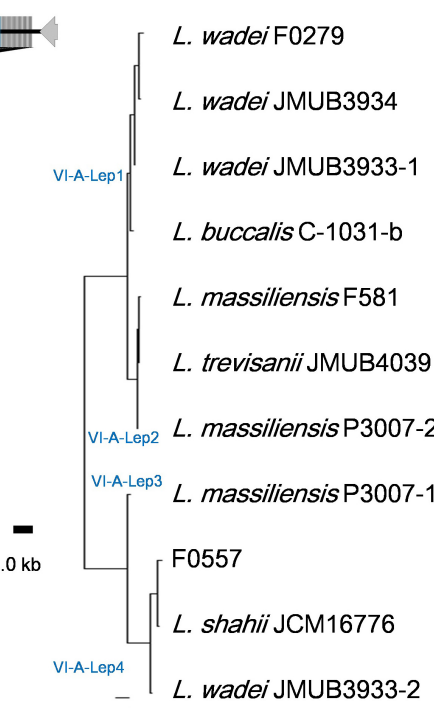

1.0

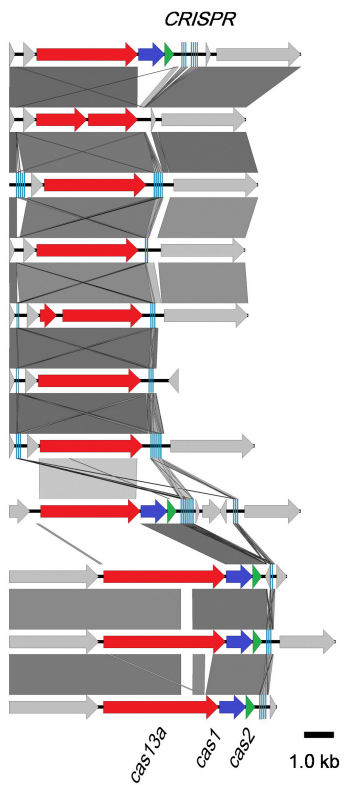

C Type III-A and -D CRISPR-cas

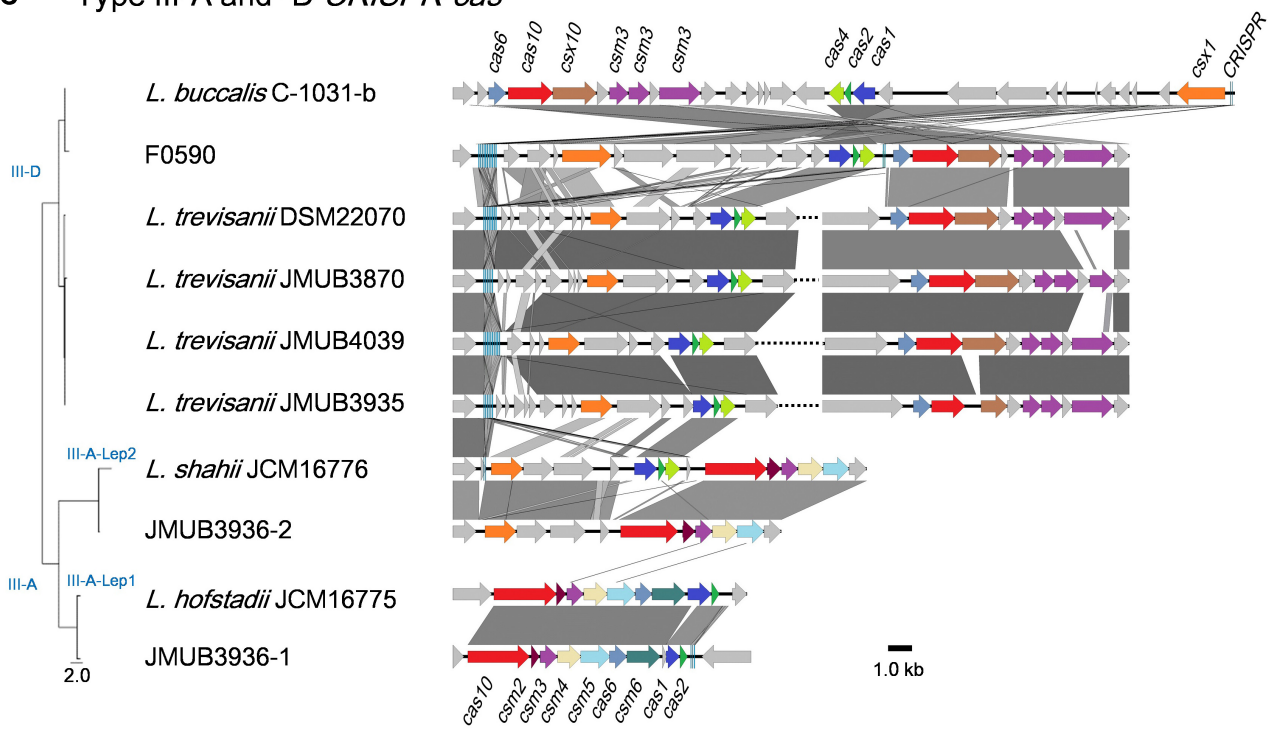

D Type III-like $C R / S P R$-cas

\section{L. sp. W10393}

L. goodfellowii JCM16774

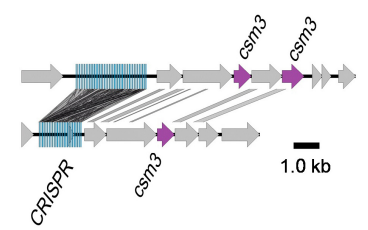

FIGURE 3 | Schematic representations of (A) Type I-B, (B) Type II-C, (C) Type III-A and -D, (D) Type III-like, and (E) Type VI-A of the CRISPR-Cas systems in Leptotrichia genomes. Genetic elements of CRISPR-Cas systems identified in this study are arranged according to their relative position in the chromosome. Different color arrows indicate different categories of cas genes: red, genes of cas8, cas9, cas 10, and cas 13a; blue, cas 1; pink, cas3; yellow, cas4; orange, cas5 and csx1; cobalt blue, cas6; brown, cas7 and csx10; dark purple, csm2; purple, csm3; light yellow, csm4; light blue, csm5; deep green, csm6; gray, other genes. Left panels at the strain names represent phylogenetic parsimony trees calculated and drawn by kSNP3 and FigTree, respectively, based on DNA sequences covering all CRISPR/cas loci. Note that the regions with homologies of $64 \%$ or more show in gray bar. 
genomes of L. goodfellowii strain JCM16774 and L. sp. W10393 (Figure 3D). However, neither of these two genomes carried type III cas genes.

The present analysis revealed that the type VI-A CRISPRcas system, which carries the single effector protein Cas13a (formerly C2c2), is broadly distributed throughout Leptotrichia species (Table 1). Eleven type VI-A CRISPR-cas loci were identified in five known and one unclassified Leptotrichia species. As shown in Figure 3E, the type VI-A loci of Leptotrichia were further classified into four subtypes, VI-ALept1 to VI-A-Lept4. Cas13a is a crRNA-guided RNA-targeting effector, which has two distinct RNase motifs (Shmakov et al., 2015; O'Connell, 2019). In addition to the single RNA-guided ssRNA cleavage activity, once activated by the cognate target RNA, Cas13a becomes a promiscuous RNase that can nonsequence-specifically cleave host cell RNA (namely collateral RNA cleavage), leading to host cell death or cellular dormancy (Abudayyeh et al., 2016; East-Seletsky et al., 2016). Previously, Cas13a loci were identified and characterized in L. wadei F0279,
L. buccalis C-1031-b and L. shahii DSM 19757, which were named as LwaCas13a, LbuCas13a and LshCas13a, respectively (Abudayyeh et al., 2016, 2017; East-Seletsky et al., 2016). In addition, Cas13a loci were identified in three Leptotrichia species in the current study, L. massiliensis, L. trevisanii and the unclassified Leptotrichia strain F0557. Genomic analysis showed that, except for the cases of L. wadei JMUB3934 and L. massiliensis F581, of which cas13a genes were truncated, all of the VI-A CRISPR Cas13a systems identified in this study were thought to have a biological function, as based on the gene structures of the three characterized systems. Moreover, the CRISPR-associated genes cas 1 and cas2, encoding an adaptation module of CRISPR-Cas13a, were usually located downstream of Cas13a in subtypes VI-A-Lep3 and VI-A-Lep4. This was also the case for the VI-A-Lep1 CRISPR-cas system of L. wadei F0279. It has been suggested that the Cas13a of VI-A-Lep3 and 4, including L. shahii JCM16776 and L. wadei JMUB3933, may be more efficient than the other subtypes because the cas $13 a$, cas 1 and cas 2 genes make up an operon

\section{A L. wadei JMUB3933 Cas13a-1}

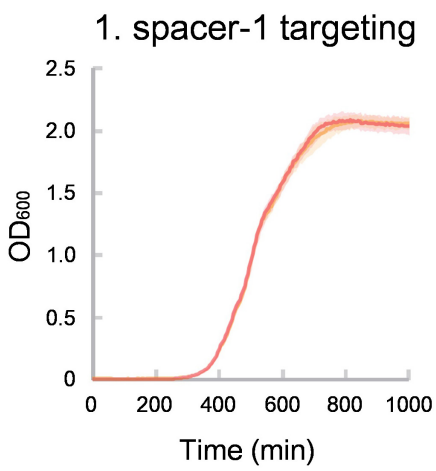

C

L. shahii JCM16776 Cas13a

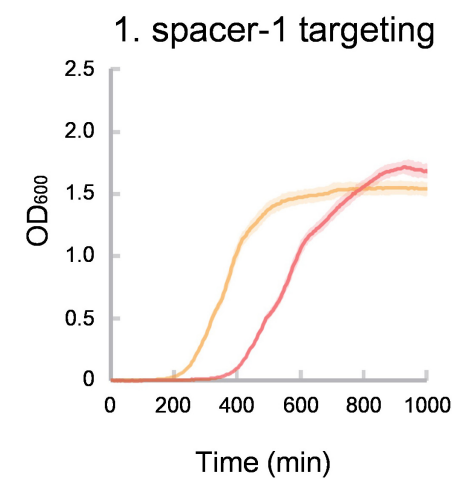

2. spacer-2 targeting

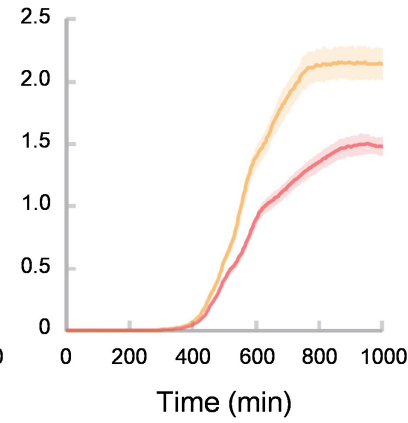

B

L. wadei JMUB3933 Cas13a-2
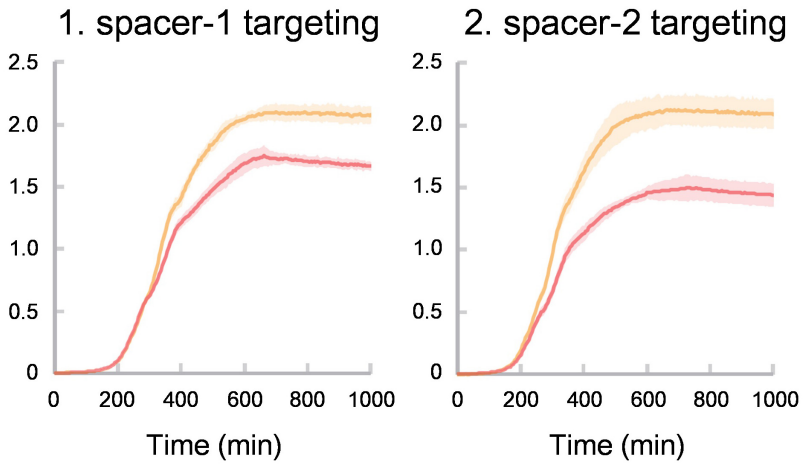

\section{L. trevisanii JMUB4039 Cas13a}
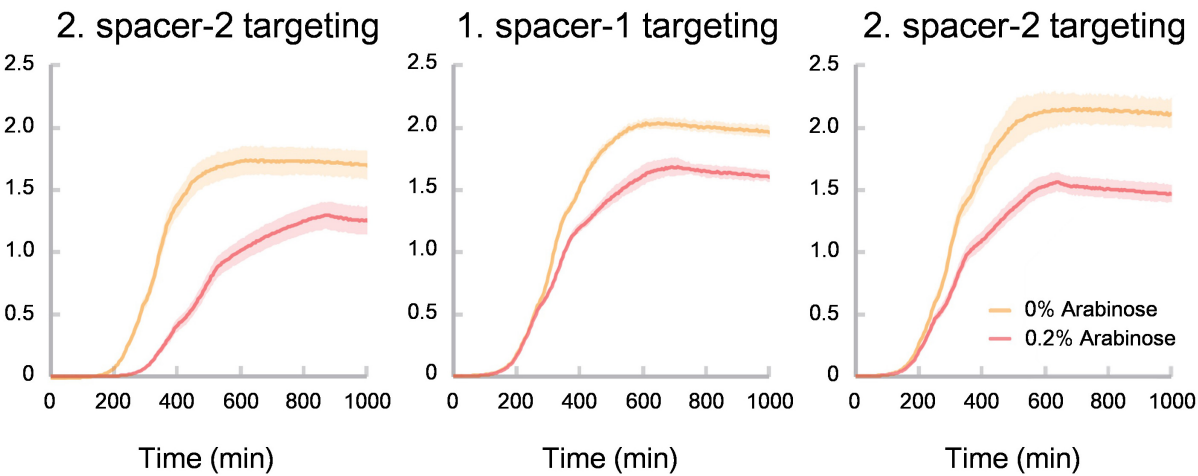

FIGURE 4 | Collateral growth inhibitory activities of (A) LwaCas13a-1, (B) LwaCas13a-2, (C) LshCas13a, and (D) LtrCas13a. Native CRISPR-Cas13a expression vector carrying kanamycin-resistant gene and arabinose-inducible target RNA (protospacer) expression vector which carries chloramphenicol-resistant gene were co-transformed into E. coli MC1061 and incubated at $37^{\circ} \mathrm{C}$ on LB plates containing chloramphenicol and kanamycin until colonies appeared. The independent colonies were transferred to LB medium containing chloramphenicol, kanamycin and $0.2 \%$ arabinose (inducer of target RNAs) and incubated with gentle shaking at $25 \mathrm{rpm}$ at $37^{\circ} \mathrm{C}$. Optical density at $600 \mathrm{~nm}(\mathrm{OD} 600)$ were measured every $5 \mathrm{~min}$ and the growth curves were obtained. Spacer sequences used here did not match against the E. coli genome with $>80 \%$ identity. 
with a structure that usually provides good spatial regulation during compensation.

Three strains of Leptotrichia have multiple loci of same types of CRISPR-Cas systems. Strain JMUB3933 and L. massiliensis P3007 carried two loci of the type VI-A system as well as L. wadei JMUB3936 had two loci of the type III-A system. In all cases of the three strains, only one of the two loci possessed cas 1 and cas 2 genes encoding spacer acquisition module. Therefore, it is suggested that the multiple CRISPRCas systems share the adaptation modules to acquire a spacer sequence into each CRISPR array. Such communal modules and CRISPR arrays are also observed in co-habiting type I systems (Makarova et al., 2011). Moreover, Leptotrichia strains frequently possess multiple CRISPR-Cas systems of different types. Our genome analysis revealed that ten of twenty nine Leptotrichia species carry more than two of types VI-A, I-B,

No. of CRISPR spacers

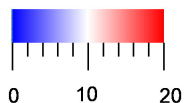

Type I-B CRISPR-cas

L. buccalis C-1031-b

L. goodfellowii JCM16774

L. shahii JCM16776

L. trevisanii DSM22070

L. trevianii JMUB3870

L. trevisanii JMUB4039

L. wadei JMUB3933

W10393
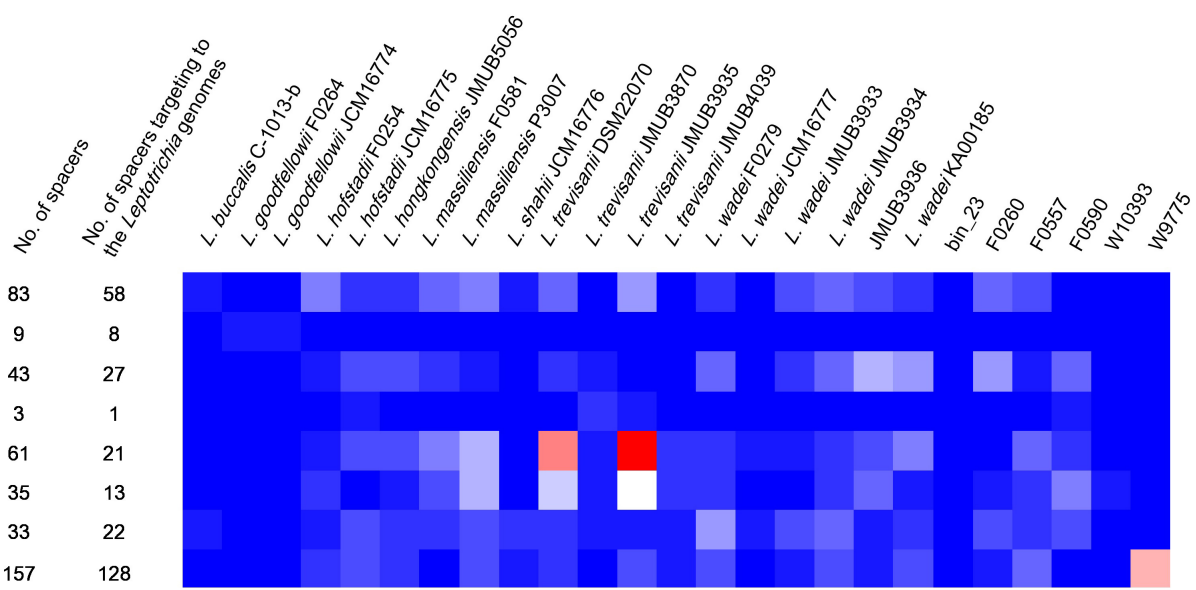

Type II-C CRISPR-cas

$\begin{array}{lll}\text { W9775 } \quad 18 & 13\end{array}$

Type III-A, -D and -like CRISPR-cas

$$
\begin{array}{r}
\text { L. buccalis C-1031-b } \\
\text { L. goodfellowii JCM16774 } \\
\text { L. shahii JCM16776 } \\
\text { L. trevisanii DSM22070 } \\
\text { L. trevisanii JMUB3870 } \\
\text { L. trevisanii JMUB3935 } \\
\text { L. trevisanii JMUB4039 } \\
\text { JMUB3936 } \\
\text { F0590 } \\
\text { W10393 }
\end{array}
$$

$\begin{array}{cc}1 & 1 \\ 25 & 21 \\ 1 & 1 \\ 7 & 2 \\ 13 & 6 \\ 7 & 7 \\ 9 & 8 \\ 2 & 2 \\ 11 & 11 \\ 42 & 34\end{array}$



Type VI-A CRISPR-cas

$\begin{array}{rll}\text { L. wuccalis } \mathrm{C}-1031-\mathrm{b} & 1 & 1 \\ \text { L. massiliensis } \mathrm{F} 581 & 3 & 3 \\ \text { L. massiliensis } \mathrm{P} 3007-1 & 8 & 7 \\ \text { L. massiliensis } \mathrm{P} 3007-2 & 7 & 7 \\ \text { L. shahii JCM16776 } & 5 & 3 \\ \text { L. trevisanii JMUB4039 } & 3 & 3 \\ \text { L. wadei } \mathrm{F} 0279 & 5 & 5 \\ \text { L. wadei JMUB3933-1 } & 8 & 3 \\ \text { L. wadei JMUB3933-2 } & 3 & 0 \\ \mathrm{~F} 0557 & 1 & 1\end{array}$

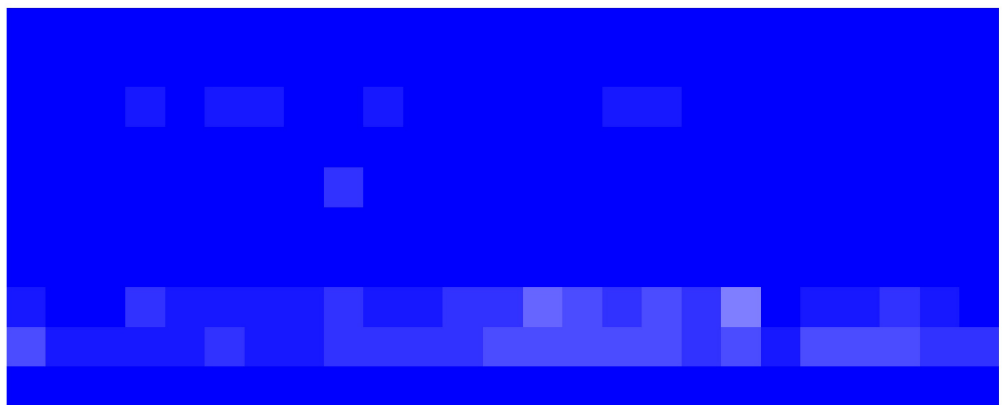

FIGURE 5 | Match of Leptotrichia CRISPR-Cas spacer sequences with sequences of phages, plasmids and bacterial genomes of own bacterial genus of Leptotrichia. This heat map shows number of spacers and the present of matched sequence with homology at least $90 \%$ in genomes of genus Leptotrichia. The first left panel indicates a total numbers of spacers present in each strain, and the second left panel does the numbers of spacer which have hit matches against each strain. 
III-A/D and III-like systems on the genomes. Similar to the cases of same type systems, different types of CRISPR-Cas systems in single Leptotrichia genome also share the adaptation modules. For example, L. trevisanii JMUB4039 carries three CRISPR-cas loci; types I-B, III-D and VI-A. Since only the type I-B locus includes adaptation module genes cas 1 and cas 2 , the other types III-D and VI-A could utilize the Cas1 and Cas2 of type I-B. These multiple CRISPR-Cas systems might be the basis for an effective defense against foreign mobile genetic elements. In addition to their canonical function in defending foreign nucleic acids, new biological functions of CRISPR-Cas systems have been recognized. In Pseudomonas aeruginosa, CRISPR-Cas system is known to be required for bacteriophage DMS3-dependent inhibition of biofilm formation (Cady and O'Toole, 2011). While in Porphyromonas gingivalis, CRISPR-Cas can affect efficiency of IS transposition and genome recombination (Watanabe et al., 2013). Relationship between CRISPR-Cas and bacterial virulence has also been described in Campylobacter jejuni, Neisseria meningitides, and Legionella pneumoniae (Gunderson and Cianciotto, 2013; Louwen et al., 2013; Sampson et al., 2013).

Of the identified CRISPR-Cas13a systems, the Cas13a effectors were highly divergent with amino acid sequence similarities ranging from $61 \%$ to $90 \%$ to that of L. shahii JCM16776 (Supplementary Table S4), which is the most intensively studied for collateral RNA cleavage activity (Abudayyeh et al., 2016). In the present study, the two most distanced CRISPR-Cas13a systems, LwaJMUB3933Cas13a1 and LwaJMUB3933Cas13a-2, were identified in L. wadei strain JMUB3933. The amino acid sequence similarities of LwaJMUB3933Cas13a-1 and LwaJMUB3933Cas13a-2 to that of L. shahii JCM16776 (LshCas13a) were 61\% and 87\%, respectively. In addition, Cas13a was firstly identified in L. trevisanii genome (L. trevisanii JMUB4039, LtrCas13a). The cytotoxic activities dependent on collateral ssRNA cleavage activity of the four systems (LwaJMUB3933Cas13a-1 and -2, LtrCas13a, LshCas13a) were compared with the use of a co-expression assay, where two different vectors carrying the CRISPR-Cas13a with spacer sequence and target sequences were co-transformed into competent E. coli cells, and then the growth curves were observed with or without $0.2 \%$ arabinose inducing mRNA which contain the target sequences. As shown in Figure 4, except for spacer-1 targeting of LwaJMUB3933Cas13a-1, the four Cas13a systems could significantly affect the growth of the host cells, indicating that the collateral cellular dormancy as a result of ssRNA cleavage were maintained among those strains. Here we demonstrated the collateral cytotoxic activities of four Cas13a using representative native spacers, a comprehensive study is necessary to draw precise conclusions regarding enzymatic activities.

CRISPR-Cas spacers represent a sequential achievement of former intruder encounters, and the retained spacers reflect evolutionary phylogeny or relatedness of the strains. In the present study, the CRISPR spacers of all strains were analyzed, as well as the protospacers of the strains with complete whole genomes. A protospacer is a spacer homolog existing in the plasmid, phage or chromosomal DNA that matches the spacers of CRISPR elements. The number and distribution of spacers and the corresponding protospacers are summarized in Supplementary Table S5 and Figure 5. As shown in Figure 5, the number of spacers had varied among the strains, ranging from 1 to 157 , which reflects the biological diversity of Leptotrichia species that acquired a CRISPR-Cas system for mediation of immunity. Type I-B, II-C and III-like CRISPR-Cas systems generally contains higher number of spacers than type III-A, III-D, and VI-A. The protospacer distribution showed that cross-targeting events within the species were very rare, but often occurred inter-species. Notably, some spacers matched against protospacers existed in the same genome. For example, spacer-1 (ATAATGTCTCAACTTAGTTTCAGTGCACTCTA) and spacer-2 (TTTTTAAACTCATCAGCCAACCAGTCAATGA) targeting of LwaJMUB3933Cas13a-2 completely matched against $50 \mathrm{~S}$ ribosomal protein L33 gene rpmG and chaperone gene dnaK of strain JMUB3933, respectively. The CRISPR Cas13a system of LwaJMUB3933Cas13a-2 and the natural spacers could significantly affect the growth of the host E. coli cells (Figure 4D). As a possible interpretation, CRISPR-Cas systems may be regulated by unknown mechanisms that degrade their own function, as self-targeting results in persistence or cell death. In fact, some bacteria have intact CRISPR-cas genes and spacers, targeting their native chromosomal loci (Sampson and Weiss, 2014). Aklujkar et al. reported that a Pelobacter carbinolicus strain had type I CRISPR-Cas system with a spacer targeting the histidyl-tRNA synthetase gene, thereby regulating its expression (Aklujkar and Lovley, 2010). Sampson et al. (2013) also described that type II CRISPR-Cas system in Francisella novicida regulated the expression of an endogenous transcript encoding a bacterial lipoprotein. Hence, we postulated that CRISPR-Cas systems in Leptotrichia may play a role in more than just self-defense immunity.

\section{CONCLUSION}

In this study, we overviewed CRISPR-Cas systems in genus Leptotrichia and the comparative genomic analysis show that types VI-A as well as types I-B and III-A/D systems are predominantly distributed in Leptotrichia. Since most genus Leptotrichia have been found from bacteria composed of oral and gut flora in human and animal, and RNA type bacteriophages are infrequently found in gut, the RNA-targeting type VI-A system (CRISPR-Cas13a) might play an important role for bacterial surviving in the niche of oral cavity and gastrointestinal tract. However, this study showed that the VI-A system of Leptotrichia could get activated by natural spacers, including self-targeting spacers, to impede host cell growth, therefore, we postulated that CRISPR-Cas system has a more significant role other than immunity in the biology of Leptotrichia species.

\section{DATA AVAILABILITY STATEMENT}

The genome sequence has been deposited at DDBJ/GenBank: PRJDB7856 (Bioproject) and DRA007809 (Raw data). 


\section{ETHICS STATEMENT}

Ethics approval and consent to participate were not required, following the ethical guidelines for medical and health research involving human subjects by Ministry of Health, Labor and Welfare, Japan since this study analyzed bacteria which were isolated as a clinical specimen and patients' personal health information could not be accessed (http://www.mhlw.go.jp/stf/seisakunitsuite/bunya/hokabunya/k enkyujigyou/i-kenkyu/index.html). Bacterial isolates were taken from hospitals as part of the standard patient care and used anonymously.

\section{AUTHOR CONTRIBUTIONS}

SW, KK, and LC designed the study. SW, BC, KK, YA, X-ET, YS, MK, TB, KT, YT, F-YL, AA, and TS performed the experiments and analyzed the data. YN coordinated the clinical sample collection. SW, BC, and KK prepared the figures and drafted the manuscript. SW, BC, KK, X-ET, and LC edited and revised the manuscript. All authors read and approved the final manuscript.

\section{FUNDING}

This work was supported by JSPS KAKENHI (Grant Nos. $15 \mathrm{H} 05654$ and $19 \mathrm{~K} 08960$ to SW, $18 \mathrm{~K} 15149$ to KK, 17K15691 to

\section{REFERENCES}

Abudayyeh, O. O., Gootenberg, J. S., Essletzbichler, P., Han, S., Joung, J., Belanto, J. J., et al. (2017). RNA targeting with CRISPR-Cas13. Nature 10, 280-284. doi: 10.1038/nature24049

Abudayyeh, O. O., Gootenberg, J. S., Konermann, S., Joung, J., Slaymaker, I. M., Cox, D. B., et al. (2016). C2c2 is a single-component programmable RNAguided RNA-targeting CRISPR effector. Science 353:aaf5573. doi: 10.1126/ science.aaf5573

Aklujkar, M., and Lovley, D. R. (2010). Interference with histidyl-tRNA synthetase by a CRISPR spacer sequence as a factor in the evolution of Pelobacter carbinolicus. BMC Evol. Biol. 10:230. doi: 10.1186/1471-2148-10-230

Arndt, D., Grant, J. R., Marcu, A., Sajed, T., Pon, A., Liang, Y., et al. (2016). PHASTER: a better, faster version of the PHAST phage search tool. Nucleic Acids Res. 07, W16-W21. doi: 10.1093/nar/gkw387

Bhaya, D., Davison, M., and Barrangou, R. (2011). CRISPR-Cas systems in bacteria and archaea: versatile small RNAs for adaptive defense and regulation. Annu. Rev. Genet. 45, 273-297. doi: 10.1146/annurev-genet-110410-132430

Bik, E. M., Long, C. D., Armitage, G. C., Loomer, P., Emerson, J., Mongodin, E. F., et al. (2010). Bacterial diversity in the oral cavity of 10 healthy individuals. ISME J. 4, 962-974. doi: 10.1038/ismej.2010.30

Bolivar, I., Whiteson, K., Stadelmann, B., Baratti-Mayer, D., Gizard, Y., Mombelli, A., et al. (2012). Bacterial diversity in oral samples of children in niger with acute noma, acute necrotizing gingivitis, and healthy controls. PLoS Negl Trop Dis. 6:e1556. doi: 10.1371/journal.pntd.0001556

Cady, K. C., and O'Toole, G. A. (2011). Non-Identity-Mediated CRISPRBacteriophage Interaction Mediated via the Csy and Cas3 Proteins. J. Bacteriol. 193, 3433-3445. doi: 10.1128/JB.01411-10

Clarridge, J. E. III (2004). Impact of $16 \mathrm{~S}$ rRNA gene sequence analysis for identification of bacteria on clinical microbiology and infectious diseases. Clin. Microbiol. Rev. 17, 840-862. doi: 10.1128/CMR.17.4.840-862.2004

Couvin, D., Bernheim, A., Toffano-Nioche, C., Touchon, M., Michalik, J., Néron, B., et al. (2018). CRISPRCasFinder, an update of CRISRFinder, includes a
YS, $19 \mathrm{~K} 15740$ to MK, and 17K19570 to LC), the Takeda Science Foundation (SW and LC), the JSPS International Research Fellow (Grant No. 17F17713 to LC), and the Japan Agency for Medical Research and Development J-PRIDE (Grant Nos. JP17fm0208028, JP18fm0208028, and JP19fm0208028 to LC). The funders had no role in the study design, data collection and analysis, decision to publish or preparation of the manuscript.

\section{ACKNOWLEDGMENTS}

We thank Dr. Toyoko Oguri at the Juntendo University; Dr. Katsuko Okuzumi at the Dokkyo University Hospital; Dr. Aki Kawashima, Dr. Haruki Sawamura, and Dr. Akitaka Yokoyama at the Ichinomiyanishi Hospital; Dr. Noriyuki Abe at the Tenri Hospital; and Dr. Eri Morita, Dr. Yuji Hatano, and Dr. Satoru Hayashi at the Japanese Red Cross Gifu Hospital for kindly providing the Leptotrichia strains. We also thank Dr. Feng Zhang at the Massachusetts Institute of Technology and Dr. Masato Suzuki for kindly gifting the plasmids.

\section{SUPPLEMENTARY MATERIAL}

The Supplementary Material for this article can be found online at: https://www.frontiersin.org/articles/10.3389/fmicb. 2019.02838/full\#supplementary-material

portable version, enhanced performance and integrates search for Cas proteins. Nucleic Acids Res. 46, W246-W251. doi: 10.1093/nar/gky425

Cox, D. B. T., Gootenberg, J. S., Abudayyeh, O. O., Franklin, B., Kellner, M. J., Joung, J., et al. (2017). RNA editing with CRISPR-Cas13. Science 11, 1019-1027. doi: 10.1126/science.aaq0180

East-Seletsky, A., O’Connell, M. R., Burstein, D., Knott, G. J., and Doudna, J. A. (2017). RNA targeting by functionally orthogonal type VI-A CRISPR-Cas enzymes. Mol. Cell 66, 373-383. doi: 10.1016/j.molcel.2017. 04.008

East-Seletsky, A., O’Connell, M. R., Knight, S. C., Burstein, D., Cate, J. H., Tjian, R., et al. (2016). Two distinct RNase activities of CRISPR-C2c2 enable guide-RNA processing and RNA detection. Nature 538, 270-273. doi: 10.1038/nature19802

Eribe, E. R., and Olsen, I. (2008). Leptotrichia species in human infections. Anaerobe 14, 131-137. doi: 10.1016/j.anaerobe.2008.04.004

Eribe, E. R., Paster, B. J., Caugant, D. A., Dewhirst, F. E., Stromberg, V. K., Lacy, G. H., et al. (2004). Genetic diversity of Leptotrichia and description of Leptotrichia goodfellowii sp. nov., Leptotrichia hofstadii sp. nov., Leptotrichia shahii sp. nov. and Leptotrichia wadei sp. nov. Int. J. Syst. Evol. Microbiol. 54, 583-592. doi: 10.1099/ijs.0.02819-0

Eribe, E. R. K., and Olsen, I. (2017). species in human infections II. J. Oral. Microbiol. 9:1368848. doi: 10.1080/20002297.2017.1368848

Foster, K., Kalter, J., Woodside, W., Terns, R. M., and Terns, M. P. (2019). The ribonuclease activity of Csm6 is required for anti-plasmid immunity by Type III-A CRISPR-Cas systems. RNA Biol. 16, 449-460. doi: 10.1080/15476286.2018. 1493334

Gardner, S. N., Slezak, T., and Hall, B. G. (2015). kSNP3.0: SNP detection and phylogenetic analysis of genomes without genome alignment or reference genome. Bioinformatics 31, 2877-2878. doi: 10.1093/bioinformatics/btv271

Gootenberg, J. S., Abudayyeh, O. O., Lee, J. W., Essletzbichler, P., Dy, A. J., Joung, J., et al. (2017). Nucleic acid detection with CRISPR-Cas13a/C2c2. Science 356, 438-442. doi: 10.1126/science.aam9321

Goris, J., Konstantinidis, K. T., Klappenbach, J. A., Coenye, T., Vandamme, P., and Tiedje, J. M. (2007). DNA-DNA hybridization values and their relationship 
to whole-genome sequence similarities. Int. J. Syst. Evol. Microbiol. 57, 81-91. doi: 10.1099/ijs.0.64483-0

Gunderson, F. F., and Cianciotto, N. P. (2013). The CRISPR-associated gene cas 2 of Legionella pneumophila is required for intracellular infection of amoebae. mBio 4:e0074-13. doi: 10.1128/mBio.00074-13

Gupta, R. S., and Sethi, M. (2014). Phylogeny and molecular signatures for the phylum Fusobacteria and its distinct subclades. Anaerobe 28, 182-198. doi: 10.1016/j.anaerobe.2014.06.007

Haft, D. H., Selengut, J., Mongodin, E. F., and Nelson, K. E. (2005). A guild of 45 CRISPR-associated (Cas) protein families and multiple CRISPR/Cas subtypes exist in prokaryotic genomes. PLoS Comput. Biol. 1:e60. doi: 10.1371/journal. pcbi.0010060

Han, W., Pan, S., López-Méndez, B., Montoya, G., and She, Q. (2017). Allosteric regulation of Csx1, a type IIIB-associated CARF domain ribonuclease by RNAs carrying a tetraadenylate tail. Nucleic Acids Res. 45, 10740-10750. doi: 10.1093/ nar/gkx726

Hunt, M., Silva, N. D., Otto, T. D., Parkhill, J., Keane, J. A., and Harris, S. R. (2015). Circlator: automated circularization of genome assemblies using long sequencing reads. Genome Biol. 16:294. doi: 10.1186/s13059-015-0849-0

Ivanova, N., Gronow, S., Lapidus, A., Copeland, A., Glavina Del Rio, T., Nolan, M., et al. (2009). Complete genome sequence of Leptotrichia buccalis type strain (C-1013-b). Stand. Genomic Sci. 1, 126-132. doi: 10.4056/sigs.1854

Jain, C., Rodriguez-R, L. M., Phillippy, A. M., Konstantinidis, K. T., and Aluru, S. (2018). High throughput ANI analysis of $90 \mathrm{~K}$ prokaryotic genomes reveals clear species boundaries. Nat. Commun. 9:5114. doi: 10.1038/s41467-018-07641-9

Kong, H. H., Oh, J., Deming, C., Conlan, S., Grice, E. A., Beatson, M. A., et al. (2012). Temporal shifts in the skin microbiome associated with disease flares and treatment in children with atopic dermatitis. Genome Res. 22, 850-859. doi: $10.1101 /$ gr.131029.111

Konstantinidis, K. T., and Tiedje, J. M. (2005). Genomic insights that advance the species definition for prokaryotes. Proc. Natl. Acad. Sci. U.S.A. 102, 2567-2572. doi: 10.1073/pnas.0409727102

Koren, S., Walenz, B. P., Berlin, K., Miller, J. R., Bergman, N. H., and Phillippy, A. M. (2017). Canu: scalable and accurate long-read assembly via adaptive. Genome Res. 27, 722-736. doi: 10.1101/gr.215 087.116

Loman, N. J., Quick, J., and Simpson, J. T. (2015). A complete bacterial genome assembled de novo using only nanopore sequencing data. Nat. Methods 12, 733-735. doi: 10.1038/nmeth.3444

Louwen, R., Horst-Kreft, D., de Boer, A. G., van der Graaf, L., de Knegt, G., Hamersma, M., et al. (2013). A novel link between Campylobacter jejuni bacteriophage defence, virulence and Guillain-Barre syndrome. Eur. J. Clin. Microbiol. Infect. Dis. 32, 207-226. doi: 10.1007/s10096-0121733-4

Makarova, K. S., Haft, D. H., Barrangou, R., Brouns, S. J., Charpentier, E., Horvath, P., et al. (2011). Evolution and classification of the CRISPRCas systems. Nat. Rev. Microbiol. 9, 467-477. doi: 10.1038/nrmicro 2577

Makarova, K. S., Wolf, Y. I., Alkhnbashi, O. S., Costa, F., Shah, S. A., Saunders, S. J., et al. (2015). An updated evolutionary classification of CRISPR-Cas systems. Nat. Rev. Microbiol. 13, 722-736. doi: 10.1038/nrmicro3569

Makarova, K. S., Wolf, Y. I., and Koonin, E. V. (2018). Classification and nomenclature of CRISPR-cas systems: where from here? CRISPR J. 1, 325-336. doi: 10.1089/crispr.2018.0033

Marraffini, L. A. (2015). CRISPR-Cas immunity in prokaryotes. Nature 526, 55-61. doi: $10.1038 /$ nature 15386

Munson, M. A., Banerjee, A., Watson, T. F., and Wade, W. G. (2004). Molecular analysis of the microflora associated with dental caries. J. Clin. Microbiol. 42, 3023-3029. doi: 10.1128/JCM.42.7.3023-3029.2004

Niewoehner, O., and Jinek, M. (2016). Structural basis for the endoribonuclease activity of the type III-A CRISPR-associated protein Csm6. RNA. 22, 318-329. doi: 10.1261/rna.054098.115

O'Connell, M. R. (2019). Molecular mechanisms of RNA targeting by Cas13containing Type VI CRISPR-Cas systems. J. Mol. Biol. 431, 66-87. doi: 10.1016/ j.jmb.2018.06.029
Sampson, T. R., Saroj, S. D., Llewellyn, A. C., Tzeng, Y. L., and Weiss, D. S. (2013). A CRISPR/Cas system mediates bacterial innate immune evasion and virulence. Nature 497, 254-257. doi: 10.1038/nature12048

Sampson, T. R., and Weiss, D. S. (2014). CRISPR-Cas systems: new players in gene regulation and bacterial physiology. Front. Cell. Infect. Microbiol. 4:37. doi: $10.3389 /$ fcimb.2014.00037

Seemann, T. (2014). Prokka: rapid prokaryotic genome annotation. Bioinformatics 30, 2068-2069. doi: 10.1093/bioinformatics/btu153

Shah, S. A., Alkhnbashi, O. S., Behler, J., Han, W., She, Q., Hess, W. R., et al. (2019). Comprehensive search for accessory proteins encoded with archaeal and bacterial type III CRISPR-cas gene cassettes reveals 39 new cas gene families. RNA Biol. 16, 530-542. doi: 10.1080/15476286.2018.1483685

Sheppard, N. F., Glover, C. V., Terns, R. M., and Terns, M. P. (2016). The CRISPR-associated Csx1 protein of Pyrococcus furiosus is an adenosinespecific endoribonuclease. RNA 22, 216-224. doi: 10.1261/rna.0398 42.113

Shmakov, S., Abudayyeh, O. O., Makarova, K. S., Wolf, Y. I., Gootenberg, J. S., Semenova, E., et al. (2015). Discovery and functional characterization of diverse class 2 CRISPR-Cas systems. Mol. Cell 60, 385-397. doi: 10.1016/j.molcel.2015. 10.008

Shmakov, S. A., Makarova, K. S., Wolf, Y. I., Severinov, K. V., and Koonin, E. V. (2018). Systematic prediction of genes functionally linked to CRISPRCas systems by gene neighborhood analysis. Proc. Natl. Acad. Sci. U.S.A. 115, E5307-E5316. doi: 10.1073/pnas.1803440115

Sullivan, M. J., Petty, N. K., and Beatson, S. A. (2011). Easyfig: a genome comparison visualizer. Bioinformatics 27, 1009-1010. doi: 10.1093/bioinformatics/btr039

Täubel, M., Rintala, H., Pitkäranta, M., Paulin, L., Laitinen, S., Pekkanen, J., et al. (2009). The occupant as a source of house dust bacteria. J. Allergy Clin. Immunol. 124, 834-840. doi: 10.1016/j.jaci.2009.07.045

Vestergaard, G., Garrett, R. A., and Shah, S. A. (2014). CRISPR adaptive immune systems of Archaea. RNA Biol. 11, 156-167. doi: 10.4161/rna. 27990

Walker, B. J., Abeel, T., Shea, T., Priest, M., Abouelliel, A., Sakthikumar, S., et al. (2014). Pilon: an integrated tool for comprehensive microbial variant detection and genome assembly improvement. PLoS One 9:e112963. doi: 10.1371/journal. pone. 0112963

Watanabe, S., Aiba, Y., Tan, X. E., Li, F. Y., Boonsiri, T., Thitiananpakorn, K. et al. (2018). Complete genome sequencing of three human clinical isolates of Staphylococcus caprae reveals virulence factors similar to those of $S$. epidermidis and S. capitis. BMC Genomics 19:810. doi: 10.1186/s12864-018-5185-9

Watanabe, S., Sasahara, T., Arai, N., Sasaki, K., Aiba, Y., Sato'o, Y., et al. (2016). Complete genome sequence of Streptococcus pyogenes strain JMUB1235 isolated from an acute Phlegmonous gastritis patient. Genome Announc. 4:e1133-16. doi: 10.1128/genomeA.01133-16

Watanabe, T., Nozawa, T., Aikawa, C., Amano, A., Maruyama, F., and Nakagawa, I. (2013). CRISPR regulation of intraspecies diversification by limiting IS transposition and intercellular recombination. Genome Biol. Evol. 5, 1099-1114. doi: 10.1093/gbe/evt075

Woo, P. C., Wong, S. S., Teng, J. L., Leung, K. W., Ngan, A. H., Zhao, D. Q., et al. (2010). Leptotrichia hongkongensis sp. nov., a novel Leptotrichia species with the oral cavity as its natural reservoir. J Zhejiang Univ. Sci. B. 11, 391-401. doi: 10.1631/jzus.B1000056

Conflict of Interest: The authors declare that the research was conducted in the absence of any commercial or financial relationships that could be construed as a potential conflict of interest.

Copyright (c) 2019 Watanabe, Cui, Kiga, Aiba, Tan, Sato'o, Kawauchi, Boonsiri, Thitiananpakorn, Taki, Li, Azam, Nakada, Sasahara and Cui. This is an open-access article distributed under the terms of the Creative Commons Attribution License (CC BY). The use, distribution or reproduction in other forums is permitted, provided the original author(s) and the copyright owner(s) are credited and that the original publication in this journal is cited, in accordance with accepted academic practice. No use, distribution or reproduction is permitted which does not comply with these terms. 